\title{
Transcription factor ATF cDNA clones: an extensive family of leucine zipper proteins able to selectively form DNA-binding heterodimers
}

\author{
Tsonwin Hai, ${ }^{1}$ Fang Liu, William J. Coukos, and Michael R. Green \\ Department of Biochemistry and Molecular Biology, Harvard University, 7 Divinity Avenue, Cambridge, Massachusetts \\ 02138 USA
}

\begin{abstract}
An activating transcription factor (ATF)-binding site (consensus sequence $5^{\prime}$-GTGACGT ${ }^{\mathbf{A}} \mathbf{C}^{\mathbf{A}} \mathbf{G}^{-3^{\prime}}$ ) is a promoter element present in a wide variety of viral and cellular genes. The two best-characterized classes of genes that contain ATF sites are E1A-inducible adenoviral genes and cAMP-inducible cellular genes. Here, we report the isolation of eight ATF cDNA clones, each of which is derived from a separate gene. All ATF cDNA clones examined contain a leucine zipper motif and are significantly similar to one another only within this region. The leucine zipper region of ATF proteins is also similar to that of the AP-1/c-jun family of transcription factors, whose DNA-binding site differs from the ATF-binding site at a single position. DNA binding studies reveal two mechanisms for generating further diversity from the ATF proteins. First, some, but not all, combinations of ATF proteins form heterodimers that efficiently bind to DNA. Second, although all ATF proteins bind to the ATF site, their precise interactions with DNA differ from one another, as evidenced by methylation interference analysis. Our results help to explain how a single promoter element, an ATF site, can be present in a wide variety of promoters.
\end{abstract}

[Key Words: Transcription factor ATF; DNA-binding protein; leucine zipper]

Received October 2, 1989; revised version accepted October 24, 1989.

The promoters of eukaryotic protein-coding genes are combinations of short, cis-acting elements, which provide binding sites for sequence-specific transcription factors (for review, see McKnight and Tjian 1986; Dynan 1989|. In general, a given promoter contains several binding sites, and, conversely, a given binding site is present in different promoters.

We have been studying the transcription factor ATF, whose consensus binding site is $5^{\prime}$-GTGACGTA ${ }_{C}^{A}{ }_{G}-3^{\prime}$ (Lin and Green 1988). This sequence is present in many viral and cellular promoters (Lin and Green 1988), and DNA-binding activities that interact with this element are found in a variety of eukaryotes (Jones and Jones 1989; Lin and Green 1989). The ElA-inducible adenovirus E2, E3, and E4 promoters contain one or more ATF sites (Lee et al. 1987). For the E4 promoter, the ATF sites have been shown to be required for E1A inducibility (Lee and Green 1987). In addition to viral promoters, many cellular promoters also contain ATF sites. The best studied among them are the cAMP-inducible promoters. In these promoters, the ATF site is referred to as a CAMP response element (CRE) and is required for inducibility by cAMP (for review, see Roesler et al. 1988). However,

${ }^{1}$ Current address: Department of Genetics, Harvard Medical School, Boston, Massachusetts 02115 USA. many cellular promoters contain ATF sites and are not known to be regulated by either E1A or cAMP (Table 1). Therefore, a common cis-acting DNA sequence, the ATF-binding site, is present in promoters regulated by a variety of different agents.

These observations raise the question of whether a single ATF protein mediates all of these apparently diverse transcription responses. In a previous biochemical study (Hai et al. 1988), we provided evidence that ATF is not a single factor but, rather a family of related polypeptides. Moreover, we demonstrated that ATF polypeptides are immunologically related to the $\mathrm{AP}-1 / \mathrm{c}-j u n$ family of transcription factors. In this paper, we describe the isolation and characterization of ATF cDNA clones. The results confirm and extend the conclusions of our previous study. We show that ATF represents an extensive gene and protein family, which has amino acid similarity to the AP-1/c-jun family of transcription factors. ATF proteins bind to DNA as dimers, and they selectively form heterodimers that can bind to DNA.

Results

Isolation of multiple ATF cDNA clones

ATF cDNA clones were isolated by screening $\lambda \mathrm{gt} 11$ expression libraries with a DNA probe containing three 
Table 1. Diverse regulation of promoters containing ATF sites

\begin{tabular}{ll}
\hline Promoter & Regulation \\
\hline Viral & \\
Ad early & E1A-inducible \\
HTLVI LTR & tax-inducible \\
Cellular & \\
somatostatin, VIP & cAMP-inducible \\
HSP70 & heat-inducible \\
c-fos & serum-inducible \\
HMG-CoA reductase & cholesterol-repressible \\
DNA polymerase $\beta$ & constitutive \\
\hline
\end{tabular}

Representative examples of viral and cellular promoters that contain the ATF consensus sequence are shown. These promoters were identified in a random literature search. The primary references are not shown but are available upon request.

tandem ATF-binding sites. From this screen we obtained eight different clones, designated ATF-1 through ATF-8. To examine the DNA binding specificity of the ATF-1 through ATF- 8 proteins, we determined their ability to bind to a single ATF site, compared to three other transcription factor-binding sites: AP-1, MLTF, and E4F2. The binding sites of MLTF and, particularly AP-1, are similar to the ATF consensus sequence (see legend to Fig. 1).

As shown in Figure 1, ATF-1, ATF-2, ATF-3, ATF-4, and ATF-7 bind efficiently to a single ATF site but not to an AP-1, MLTF, or E4F2 site. ATF-5 and ATF-6 bind to the triplicated ATF site with low affinity (data not shown/ but do not bind detectably to the single ATF site. Only ATF-8 binds more efficiently to an AP- 1 site than an ATF site. Nevertheless, ATF- 8 can bind to an ATF site because it was initially isolated using a triplicated ATF site probe.

\section{Amino acid sequences of ATF CDNA clones}

ATF-1 through ATF- 6 were sequenced, revealing that each ATF clone contains a long open reading frame and that each is a partial cDNA clone. It is of particular sig- nificance (discussed in detail below) that all six ATF clones contain the leucine zipper DNA-binding motif, originally described by McKnight and colleagues (Landshulz et al. 1988). The leucine zipper motif is a region with a leucine at every seventh residue and a highly basic region immediately amino-terminal to this leucine repeat (Landshulz et al. 1988).

Comparison of the deduced amino acid sequences of ATF-1 through ATF-6 indicates that ATF proteins share significant amino acid similarity within the leucine repeat and the adjacent basic region. Outside of these regions ATF proteins are not significantly similar, based on the normalized alignment score calculated according to Doolittle (1981, 1986). As an example, Figure 2A compares the amino acid sequences of ATF-1, ATF-2, and ATF-3.

As expected, the leucine zipper portion of the ATF proteins appears to mediate DNA binding: truncated proteins (ATF-1, ATF-2, and ATF-3) that contain little more than the leucine repeat and adjacent basic region bind to DNA (see below). The observation that the ATF proteins are highly similar within the leucine zipper region is consistent with the fact that these proteins bind to the same DNA site.

A survey of the literature revealed that homologs of ATF-1 and ATF-2 have recently been cloned by other groups (Hoeffler et al. 1988; Gonzalez et al. 1989; Maekawa et al. 1989). Two cDNA clones of a protein designated cAMP response element (CRE) nuclear binding protein (CREB) have been isolated: One clone was isolated by screening a human placental expression library for DNA-binding activity to a CRE/ATF site (Hoeffler et al. 1988); the other clone was isolated by screening a rat PC12 cDNA library for hybridization to oligonucleotides synthesized on the basis of partial amino acid sequence of the purified CREB protein (Gonzalez et al. 1989). These two cDNA clones differ from each other in only two peptide regions, which may be the result of a cross-species variation or alternative splicing.

Figure 2B compares ATF-1 to the CREB sequence reported by Hoeffler et al. (1988). ATF-1 and CREB are
Figure 1. DNA binding specificities of ATF proteins. Plaque-purified recombinant phage harboring ATF-1 through ATF-8 cDNA inserts were plated, and the plaques transferred to a nitrocellulose filter. Each nitrocellulose filter was cut into four quadrants, and each quadrant probed with a ${ }^{32} \mathrm{P}$-labeled DNA fragment containing either a single ATF site, 5'-ATGACGTCAT- ${ }^{\prime}$ ', a single AP-1 site 5'-TGAGTCAG-3', a single MLTF site, 5'-CCACGTGAC-3', or a single E4F2 site, $5^{\prime}$-TTCCCACG- $3^{\prime}$. Conditions for binding the ${ }^{32}$ p-labeled DNA probe and washing filters were identical to that described for the original isolation of cDNA clones (see Materials and methods). After binding and washing, the four quadrants of each filter were realigned and exposed to Kodak X-ray films at $-80^{\circ} \mathrm{C}$ for either $1 \mathrm{hr}$ (ATF-1, ATF-2, and ATF-3) or $18 \mathrm{hr}$ (ATF-4 through ATF-7). (Left) The orientation of the probes.

\section{PROBES}
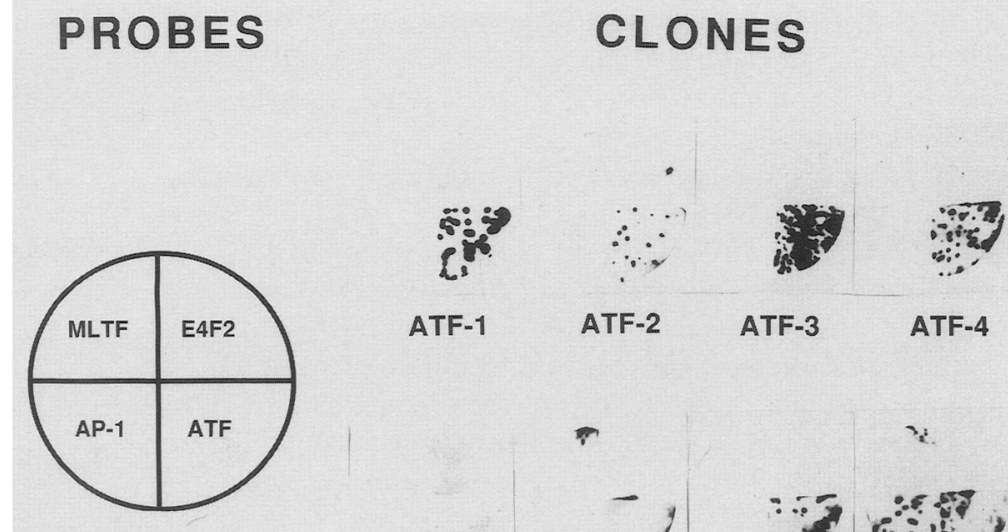

ATF-1

ATF-2

ATF-3

ATF-4

r

3
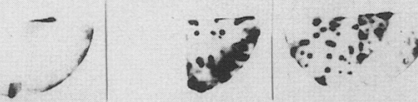

ATF-5

ATF-6

ATF-7

ATF-8 

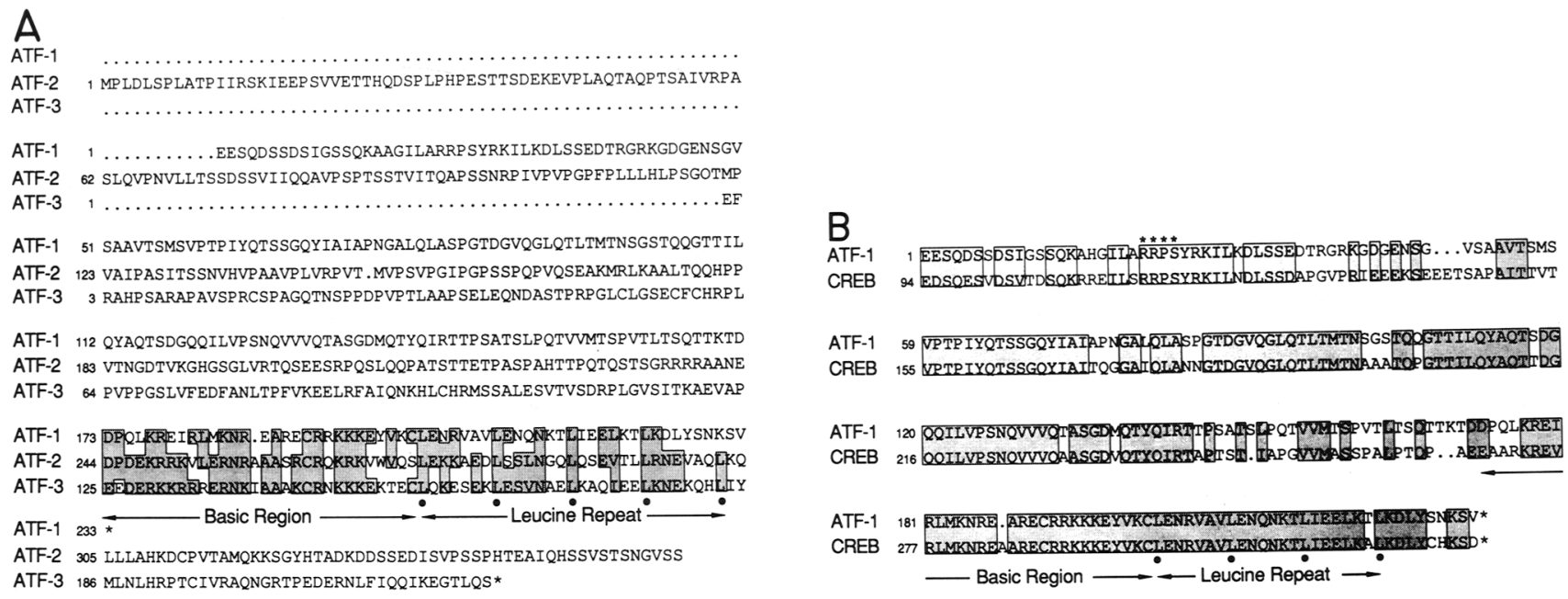

Figure 2. Sequence comparisons of ATF proteins. (A) Sequence comparison of ATF-1, ATF-2, and ATF-3. The DNA sequences are available on request. The leucine repeat and the basic region are indicated. (O) The leucines. Identical and conserved (V, L, and I; R and $\mathrm{K} ; \mathrm{E}$ and $\mathrm{D} ; \mathrm{Q}$ and $\mathrm{N}$ ) amino acids within the leucine repeat/basic region are shaded. Outside of the leucine zipper region, ATF proteins do not share significant similarity based on the alignment score (Doolittle 1981, 1986). (B) Sequence comparison of ATF-1 and CREB. The CREB amino acid sequence is taken from Hoeffler et al. (1988). The phosphorylation site for cAMP-dependent protein kinase, Arg-Arg-X-Ser, is indicated by stars. The overall amino acid similarity is $\sim 75 \%$.

clearly distinct proteins but have a $75 \%$ amino acid similarity. It has been proposed that the cAMP-inducible transcription response involves phosphorylation of CREB by protein kinase A (Yamamoto et al. 1988; Gonzalez et al. 1989). Both ATF-1 and CREB (Hoeffler et al. 1988; Gonzalez et al. 1989) contain the sequence ArgArg-X-Ser, a consensus kinase A site (Edelman et al. 1987).

A homolog of ATF-2, designated CRE-BP1, was isolated from a rat brain cDNA library (Maekawa et al. 1989|. Sequence comparison shows that the homolog is identical to ATF-2 except for 3-nucleotide differences, which change 2 amino acids. Thus, ATF- 2 is likely to be the human homolog of the rat CRE-BP1.

As described above, ATF- 6 binds weakly to the triplicated ATF site and does not bind to the single ATF site. The sequence analysis reveals that the leucine repeat of ATF-6 is disrupted: One leucine residue in ATF-6 is replaced by an alanine, and another by a valine (Fig. 3). Several studies have shown that mutations of one or more leucines in the leucine repeats can interfere with dimer formation, resulting in a lower DNA binding affinity (for review, see Abel and Maniatis 1989). Therefore, it seems likely that the relatively low binding affinity of the ATF- 6 protein is a result of its disrupted leucine repeat.

\section{ATF and AP-1 share similar DNA-binding domains}

Figure 3 compares the leucine zipper regions of ATF proteins and AP-1/c-jun proteins. ATF and AP-1/c-jun proteins share significant amino acid similarity in this region, consistent with the fact that they bind to highly related DNA sequences. The ATF consensus is $5^{\prime}$ GTGACGTA ${ }_{C}^{A}{ }_{G}-3^{\prime}$, whereas the AP-1 consensus is $5^{\prime}$ GTGAGTA ${ }_{C} A-3^{\prime}$. We have looked for features in the amino acid sequences that could explain the differential DNA binding specificities of these two protein families; as yet, no obvious sequence features distinguish these two families. Outside of their DNA-binding domains, ATF and AP-1 do not share significant similarity based on the Doolittle alignment score (Doolittle 1981, 1986).

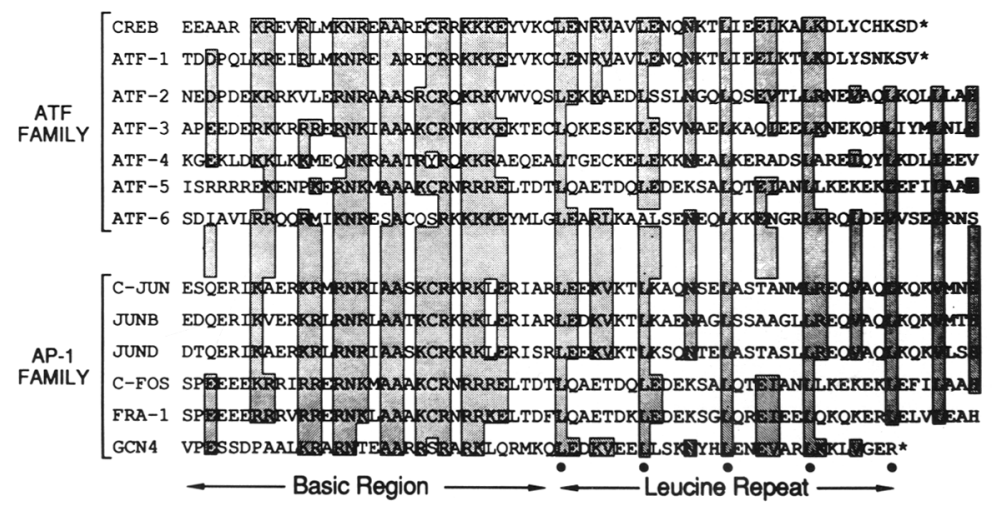

Figure 3. Sequence comparison of the DNA-binding domains of ATF and AP-1 proteins. Sequences of ATF proteins are from this report, and the sequences of the AP-1/c-jun proteins are from published studies. The DNA sequences of ATF cDNA clones are available upon request. The leucine repeat and the basic region are indicated. (1) The leucines. Identical and conserved amino acids are shaded. Only residues that are more than $50 \%$ conserved among the different proteins are indicated. 


\section{Dimethylsulfate interference analysis of ATF proteins}

We studied the DNA binding properties of three ATF proteins, ATF-1, ATF-2, and ATF-3, in further detail. First, we used dimethylsulfate (DMS) interference analysis to determine whether ATF-1, ATF-2, and ATF-3 interact with DNA differently. As a probe we used an adenovirus E4 DNA fragment that contains an ATF site and can confer E1A inducibility on heterologous promoters (Lee and Green 1987). Truncated ATF-1, ATF-2, and ATF-3 proteins containing the leucine zipper region were expressed by in vitro translation (for details, see Materials and methods). As shown in Figure 4A, methylation of guanosine 166 (G166) on the coding strand interfered with the binding of ATF-1, ATF-2, and ATF-3. Methylation of G161, however, only diminished the binding of ATF-3, and did not affect binding of ATF-1 or ATF-2. Comparable results were obtained by DMS protection analysis: ATF-3, but not ATF-1 or ATF-2, protected G161 from methylation (data not shown). These results indicate that ATF-3 makes an intimate contact with N7 of G161. In contrast, ATF-1 and ATF-2 do not interact with this chemical group. Thus, there are subtle differences in the interaction between the different ATF proteins and DNA.

DMS interference analysis on the opposite strand (mRNA-like strand) did not reveal differences in the in- teractions between ATF-1, ATF-2, and ATF-3 with DNA (Fig. 4B). Methylation of G165 and G170 decreased the binding of all three proteins. (G168, the third guanosine residue within the ATF site, is not modified well on naked DNA, therefore, we could not conclude whether modification of this residue affects binding.) The combined results of the methylation interference experiments are summarized in Figure 4C.

\section{ATF proteins bind to DNA as dimers}

The presence of leucine repeats in ATF proteins prompted us to examine whether they bind to DNA as dimers. We used an assay originally described by Hope and Struhl (1987). Proteins of different sizes (designated as large and small) were produced from the same ATF cDNA clone by in vitro translation and analyzed for DNA binding, using a mobility-shift assay. As expected, the electrophoretic mobility of the DNA-protein complex formed by the large protein is less than that formed by the small protein. When the large and small proteins were cotranslated or mixed together following translation, a third DNA-protein complex of an intermediate electrophoretic mobility appeared (Fig. 5). The intermediate complex indicates the formation of a mixed dimer between the large and small proteins (L : S dimer). Thus,
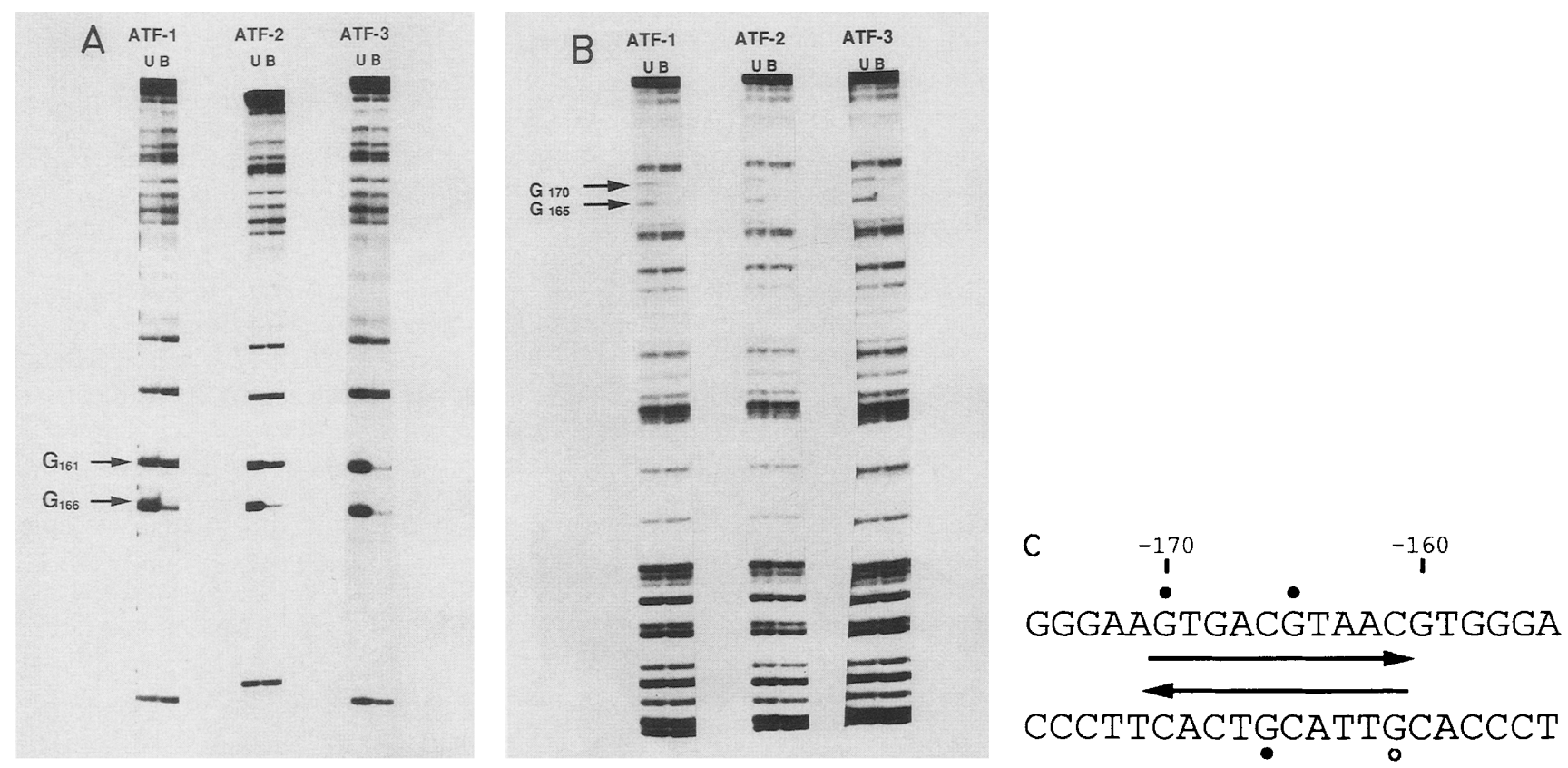

Figure 4. DMS interference analysis of ATF-1, ATF-2, and ATF-3. (A) Coding strand. A DNA fragment from approximately - 60 to -210 of Ad5 E4 promoter was [32 P]-end-labeled with reverse transcriptase and [32 P]dNTPs and treated with DMS so that, on average, one guanosine was modified per molecule. Modified DNA was incubated with in vitro-synthesized ATF-1, ATF-2, or ATF-3. Bound DNA was separated from unbound DNA on a native gel. The DNA molecules were purified from the gel and cleaved by piperidine, as described in Materials and methods. (Left) G161 and G166 within the ATF-binding site are indicated. Unbound (U) and bound (B) DNA are indicated above each lane. (B) mRNA-like strand. (Left) G165 and G170 within the ATF-binding site are indicated. G168 is not efficiently modified by DMS, perhaps due to a local secondary or tertiary structure of the naked DNA that makes N7 of G168 less accessible. (C) Summary of DMS interference analysis. The DNA sequence from -155 to -175 of the adenovirus E4 promoter is shown. The ATF site is imperfectly symmetric, as indicated by the arrows. (O) The guanosine residues contacted by ATF-1, ATF-2, and ATF-3; $(O)$ the guanosine residue contacted only by ATF-3. 


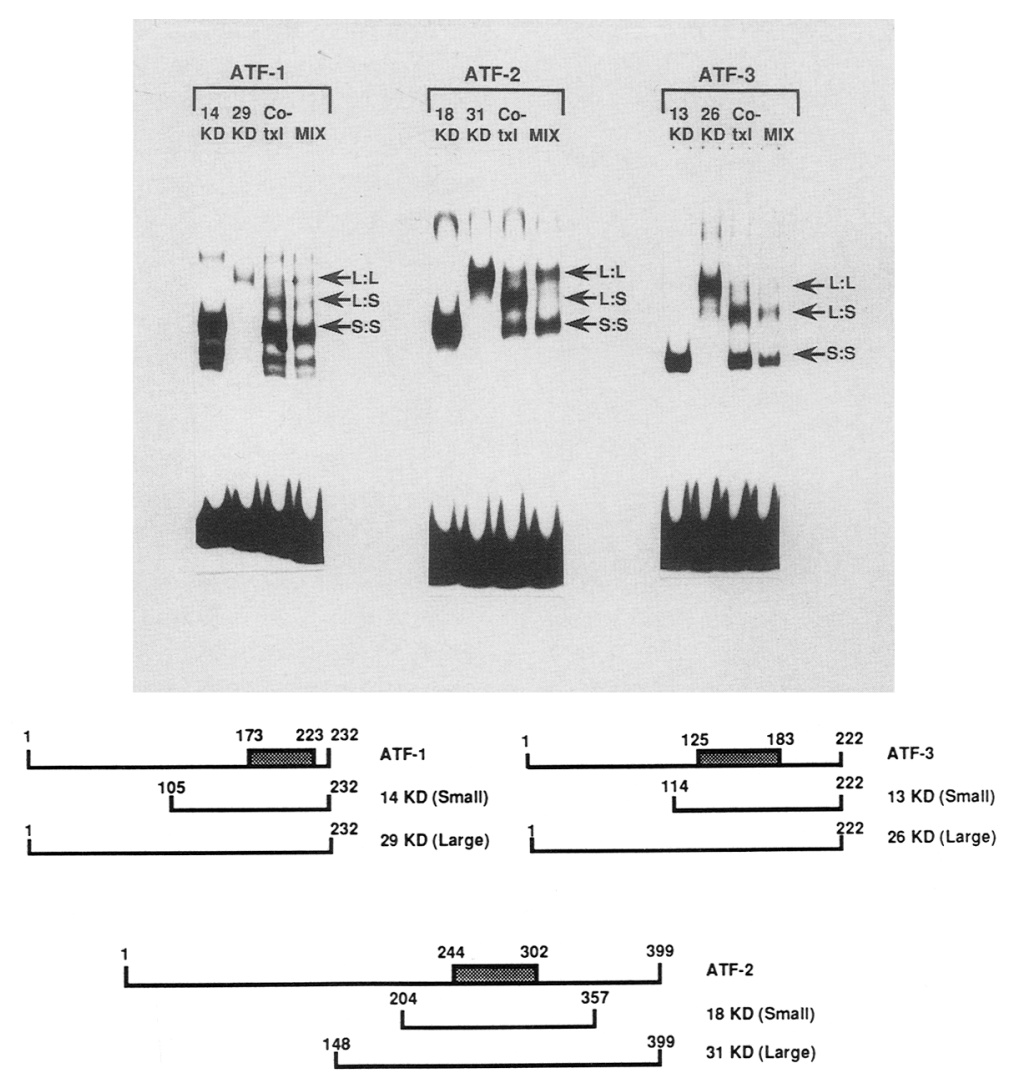

Figure 5. ATF-1, ATF-2, and ATF-3 bind to DNA as dimers. Proteins of different sizes, designated large (L) or small (S), were synthesized from the same ATF cDNA clone by in vitro translation. The predicted molecular mass $(\mathrm{kD})$ of each protein is indicated above the lane. Binding of in vitro-synthesized proteins to a ${ }^{32} \mathrm{P}$-labeled DNA probe was analyzed using a mobility-shift assay. The proteins present in the DNA binding reaction are indicated above the lanes. (Co-txl); Cotranslation of the two proteins; (MIX) mixing of the proteins following synthesis. The compositions of the DNA-protein complexes are indicated at right. Homodimer formation is evidenced by the appearance of an intermediate complex $(\mathrm{L}: \mathrm{S})$ when proteins of different sizes were cotranslated or mixed following synthesis. Schematic diagrams of the truncated ATF-1, ATF-2, and ATF-3 are shown at bottom. Relevant amino acid numbers are indicated at top of each line. Amino acid number 1 is the first amino acid derived from the partial cDNA clone. (Shaded box); Leucine zipper region. The approximate molecular mass of each truncated protein $(\mathrm{kD})$ is indicated at right.

ATF-1, ATF-2, and ATF-3 all bind to DNA as dimers. In each instance, formation of the $\mathrm{L}: \mathrm{S}$ dimer is greater when the large and small proteins are cotranslated than when they are mixed together following translation. This is presumably because the preformed $\mathrm{L}: \mathrm{L}$ and $\mathrm{S}: \mathrm{S}$ dimers are relatively stable.

\section{Selective formation of DNA-binding heterodimers}

We investigated whether ATF proteins can form heterodimers that bind to an ATF site. An experiment like the one shown in Figure 5 was performed, except that the large and small ATF proteins were synthesized from different ATF cDNA clones. The results shown in Figure 6 indicate that ATF-2 and ATF-3 are able to form a heterodimer that binds to DNA. In contrast, ATF-1 does not form a DNA-binding heterodimer with either ATF-2 or ATF-3. Therefore, our data indicate that ATF proteins are capable of forming heterodimers and that functional heterodimer formation is selective.

\section{Discussion}

\section{ATF family of transcription factors}

By screening $\lambda$ gt 11 libraries, we have isolated multiple cDNA clones whose protein products can bind to ATF sites. Therefore, as predicted from biochemical experiments (Hai et al. 1988), ATF is a family of transcription factors. Each member of the ATF family can bind to the same DNA sequence, has a similar DNA-binding domain, and is encoded by a different gene. It is likely that the size of the ATF family is significantly larger than the eight members reported here. We have not exhaustively screened libraries: Only two of the nine positive clones were identical. Furthermore, we did not isolate one member of the ATF family, designated CREB (Hoeffler et al. 1988; Gonzalez et al. 1989).

Why are there so many different ATF proteins and what are their functions? Although we can only speculate, it seems likely that the size of the ATF family is related to the apparent diversity in the regulation of pro- 


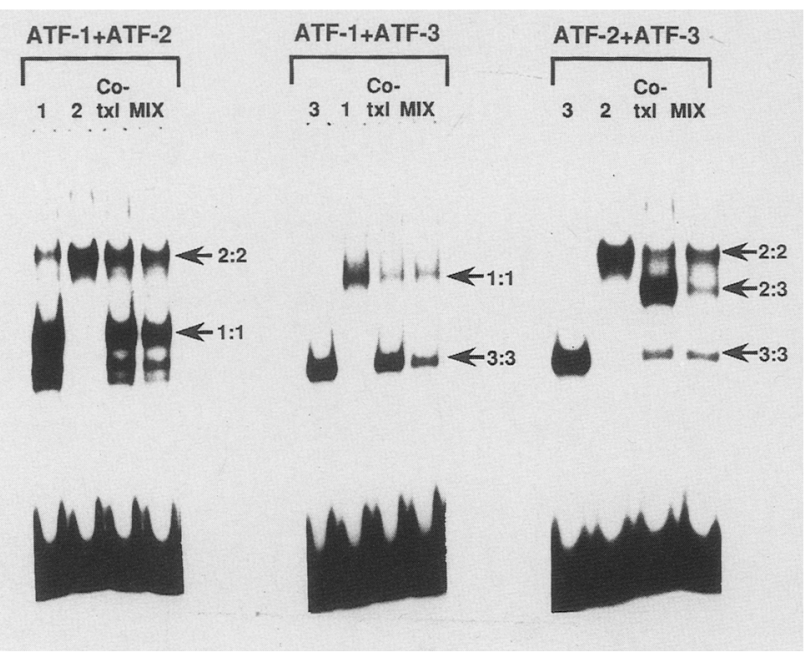

Figure 6. ATF proteins can selectively form heterodimers that bind to DNA. Proteins of distinguishable sizes were synthesized from the different ATF cDNA clones by in vitro translation. Binding of the in vitro-synthesized proteins to a ${ }^{32} \mathrm{P}-\mathrm{la}$ beled DNA probe was analyzed using a mobility-shift assay. The proteins present in the DNA binding reaction are indicated above the lanes. (Co-txl); Cotranslation of the two proteins; (MIX) Mixing of the proteins following synthesis. The compositions of the DNA-protein complexes are indicated at right. Heterodimer formation is evidenced by the appearance of an intermediate complex when proteins of different sizes were cotranslated or mixed following synthesis.

moters containing ATF sites (Table 1). These promoters can be regulated by CAMP, ElA, and a variety of other signals. It thus appears that the ATF sites within these promoters are not functionally equivalent. This notion is supported by a recent study comparing the adenovirus E4 and cellular VIP promoters. Despite the fact that both promoters contain ATF sites, the E4 promoter is highly inducible by E1A and poorly inducible by cAMP, whereas the VIP promoter is highly inducible by cAMP and poorly inducible by ElA (Lee et al. 1989). This functional dissimilarity of ATF sites could be attributable to differences in the surrounding transcription factor binding sites, or to differences in the nucleotides directly flanking the ATF sites. For example, some single base substitutions surrounding ATF sites affect inducibility by cAMP (Deutsch et al. 1988).

We speculate that the different ATF proteins bind selectively to ATF sites present in various promoters in vivo. Our methylation interference data show that the precise interactions between the various ATF proteins and DNA differ, providing at least one basis for selective binding. Once bound to the promoter, an ATF protein can mediate a particular transcription response. Outside of the DNA-binding domain ATF proteins are highly divergent. The transcriptional effector function is presumably located within these dissimilar regions. For example, the kinase A site in CREB and ATF-1 is outside of the DNA-binding domain.
The large number of ATF proteins may also play a role in tissue-specific or developmental gene expression. ATF-2 (CRE-BP1) is expressed at various levels in many tissues (Maekawa et al. 1989). We know that at least some cell lines simultaneously express several ATF genes because we have isolated multiple ATF cDNA clones from a single cDNA library.

\section{Selective formation of heterodimers that can bind to DNA}

Formation of ATF heterodimers provides an additional mechanism for generating diversity. The function of a heterodimer may differ qualitatively or quantitatively from either of the two homodimers. All of the ATF homodimers bind to an ATF site, indicating that each protein contains a DNA-binding domain able to recognize an ATF site; however, only some combinations of ATF proteins can form heterodimers that bind to DNA. This specificity can result from selective formation of heterodimers or from promiscuous formation of heterodimers whose DNA-binding activities differ. Studies of the leucine zipper proteins Jun and Fos indicate that the first possibility is more likely to be correct. Mutagenesis experiments indicate that the leucine repeat and basic region are functionally separable; the leucine repeat mediates dimerization whereas the basic region binds DNA (Gentz et al. 1989; Landschulz et al. 1989; Turner and Tjian 1989). Importantly, dimerization via leucine repeats is highly selective and is required for DNA binding. For example, Jun can dimerize with either Jun or Fos and can bind to DNA as a homodimer or a heterodimer. In contrast, Fos is unable to form a homodimer and thus cannot bind to DNA in the absence of Jun (for review, see Abel and Maniatis 1989). On the basis of this precedent, we believe that nonpromiscuous association between different ATF proteins is a likely basis for selectively generating heterodimers that can bind to DNA.

In summary, this report describes three potential mechanisms for the differential regulation of promoters that contain ATF sites: There are multiple ATF proteins, their precise interactions with DNA differ, and they selectively form functional heterodimers.

\section{Transcription factor families}

In addition to the ATF family, a number of other transcription factor families have been identified, such as the AP-1/c-jun, steroid hormone receptor and CCAAT box families. In fact, some transcription factors that were originally believed to be a single species have been shown to comprise multiple polypeptides upon further examination (e.g., see, Sawadogo et al. 1988). Thus, the phenomenon of multiple factors that bind to the same DNA site appears to be common in higher eukaryotes.

\section{Materials and methods}

Isolation of ATF cDNA clones

A $\lambda$ gt1l cDNA library prepared from HeLa cell mRNA /a gift from P. Angel and M. Karin) or from human osteosarcoma 
MG63 cell mRNA (Clontech) was screened for binding to a triplicated ATF-binding site, according to Vinson et al. (1988). A self-complementary oligonucleotide 5'-ATGACGTCAT-3', containing the ATF core consensus sequence was cloned into the HincII site of pGEM3. A DNA fragment containing three tandem repeats of the oligonucleotide was isolated from the recombinant plasmid, 3 '-end-labeled using reverse transcriptase and $\left[{ }^{32} \mathrm{P}\right] \mathrm{dNTPs}$, and used as a probe. ATF-3, ATF-5, and ATF- 6 were isolated from $\sim 1.5 \times 10^{5}$ plaques of the HeLa cell cDNA library. All other ATF cDNA clones were isolated from $\sim 3 \times 10^{5}$ plaques of an MG63 cell cDNA library.

\section{Characterization and sequencing of ATF clones}

Inserts from eight positive clones were subcloned into pGEM3. Relationships between the clones were initially analyzed by cross hybridization and restriction mapping and confirmed by dideoxy sequencing analysis. Two of the original eight subclones were identical, leaving a total of at most eight different clones. Computer-generated amino acid comparisons were based on the method of Needleman and Wunsch (1970).

\section{In vitro transcription and translation}

For in vitro transcription and translation experiments, plasmids containing truncated ATF cDNAs were constructed by fusing appropriate DNA fragments in-frame with ATG initiation codons. RNA was synthesized in a $50-\mu 1$ reaction containing 40 $\mathrm{mM}$ Tris- $\mathrm{HCl}$ (pH 7.9), $6 \mathrm{~mm} \mathrm{MgCl}_{2}, 2 \mathrm{~mm}$ spermidine, $10 \mathrm{~mm}$ DTT, $1 \mathrm{~mm}$ CTP, $1 \mathrm{~mm} \mathrm{UTP,} 1 \mathrm{~mm}$ ATP, $0.1 \mathrm{~mm}$ GTP, 1 unit of GpppG (Pharmacia), $2 \mu \mathrm{l}$ of RNasin (Promega), $5 \mu \mathrm{g}$ of linearized DNA and 40 units of T7 or SP6 RNA polymerase for $2 \mathrm{hr}$ at $40^{\circ} \mathrm{C}$. In vitro translation of the purified RNA was carried out by incubating one-tenth of the RNA in a 5- $\mu$ l reaction containing a $20 \mu \mathrm{M}$ concentration of a 20 -amino acid mixture, 2 mM DTT, $0.1 \mu l$ of RNasin, and $3.5 \mu l$ of micrococcal nucleasetreated rabbit reticulocyte lysate (Promega) at $30^{\circ} \mathrm{C}$ for $1 \mathrm{hr}$, as recommended by the manufacturer. DNA binding was assayed immediately after synthesis of the ATF proteins.

\section{DNA binding assay}

A HindIII-EcoRI DNA fragment containing the oligonucleotide 5'-ATGACGTCAT-3' and the flanking polylinker sites was isolated, 3'-end-labeled using reverse transcriptase and $\left[{ }^{22} \mathrm{P}\right] \mathrm{dNTPs}$, and used as a probe in the binding assay. The binding reaction mixture $(20 \mu \mathrm{l})$ contains $2 \mu \mathrm{l}$ of the in vitro translation reaction mixture, $2 \mathrm{nM}$ probe, $0.1 \mu \mathrm{g}$ of poly[d(I-C], and $0.5 \times$ Buffer D [10 mM HEPES (pH 8), 10\% glycerol, $50 \mathrm{~mm}$ $\mathrm{KCl}, 0.1 \mathrm{~mm}$ EDTA, and $0.25 \mathrm{~mm}$ DTT]. The reaction was carried out in two stages. All components except the probe were mixed together and preincubated at room temperature for 5 min. Following addition of the probe, incubation was continued for an additional $20 \mathrm{~min}$. The reaction was terminated by loading on a $5 \%$ (40:1 cross-linking ratio) native polyacrylamide gel containing $0.5 \times$ TBE $[45 \mathrm{~mm}$ Tris-borate, $45 \mathrm{~mm}$ boric acid and $1 \mathrm{mM}$ EDTA $\{\mathrm{pH} 8.3)]$. The gel was subjected to electrophoresis in $0.5 \times \mathrm{TBE}$ at $10 \mathrm{~V} / \mathrm{cm}$ for $2 \mathrm{hr}$ before loading and for $3.5 \mathrm{hr}$ after loading in the cold room.

\section{DMS interference analysis}

An adenovirus type 5 (Ad5 E4) promoter DNA fragment containing sequences from -210 to -60 was $3^{\prime}$-end-labeled using reverse transcriptase and $\left[{ }^{32} \mathrm{P} \mid \mathrm{dNTPs}\right.$. Then, $100 \mathrm{ng}$ of the probe was methylated in a $200-\mu \mathrm{l}$ reaction containing $50 \mathrm{~mm}$ sodium cacodylate (pH 8), 1 mM EDTA, and $1 \mu$ of DMS at $23^{\circ} \mathrm{C}$ for 4.5 $\mathrm{min}$. The reaction was terminated by the addition of $50 \mu \mathrm{l}$ of stop solution [1.5 $\mathrm{M}$ sodium acetate $(\mathrm{pH} 7), 1 \mathrm{M}$ mercaptoethanol, and $100 \mu \mathrm{g} / \mathrm{ml}$ tRNA] and $750 \mu \mathrm{l}$ of ethanol. DNA was ethanol precipitated twice prior to incubation with $16 \mu \mathrm{l}$ of in vitro-translated ATF proteins in an $80-\mu$ l reaction containing $0.8-\mu \mathrm{g}$ of poly $[\mathrm{d}(\mathrm{I}-\mathrm{C}]$ and $0.5 \times$ buffer $\mathrm{D}$. Bound and unbound DNA were separated on a $5 \%$ native polyacrylamide gel. The DNA was eluted from the gel, deproteinized, ethanol precipitated and cleaved in $100 \mu \mathrm{l}$ of $1 \mathrm{M}$ piperidine at $90^{\circ} \mathrm{C}$ for 30 min. The cleaved DNA was lyophilized and analyzed on a $10 \%$ sequencing gel.

\section{Acknowledgments}

We thank Peter Angel and Michael Karin for the $\lambda g t 11 \mathrm{HeLa}$ cell cDNA library, Steve McKnight for advice on screening expression libraries, Clair Kelley for her contribution to the DNA sequencing, and Kathy Martin for critical reading of the manuscript. T. Hai is supported by a postdoctoral fellowship from the National Institutes of Health. This work was supported by a grant from the National Institutes of Health to M.R.G.

\section{References}

Abel, T. and T. Maniatis. 1989. Action of leucine zippers. $\mathrm{Na}$ ture 341: 24-25.

Deutsch, P.J., J.P. Hoeffler, J.L. Jameson, J.C. Lin, and J.F. Habener. 1988. Structural determinants for transcriptional activation by cAMP-responsive DNA elements. J. Biol. Chem. 263: $18466-18472$

Doolittle, R.F. 1981. Similar amino acid sequences: Chance or common ancestry? Science 214: 149-159.

-1986. Of Urfs and Orfs: A primer on how to analyze derived amino acid sequences. University Science Books, Mill Valley, California.

Dynan, W.S. 1989. Modularity in promoters and enhancers. Cell 58: 1-4.

Edelman, A.M., D.K. Blumenthal, and E.G. Krebs. 1987. Protein serine/threonine kinase. Annu. Rev. Biochem. 56: 567-613.

Gentz, R., F.J. Rauscher, C. Abate, and T. Curran. 1989. Parallel association of fos and jun leucine zippers juxtaposes DNAbinding domains. Science 243: 1695-1699.

Gonzalez, G.A., K.K. Yamamoto, W.H. Fischer, D. Karr, P. Menzel, W. Biggs, W.W. Vale, and M.R. Montminy. 1989. A cluster of phosphorylation sites on the cyclic-AMP regulated nuclear factor CREB predicted by its sequence. Nature 337: 749-752.

Hai, T., F. Liu, E.A. Allegretto, M. Karin, and M.R. Green. 1988. A family of immunologically related transcription factors that includes multiple forms of ATF and AP-1. Genes Dev. 2: 1216-1226.

Hoeffler, J.P., T.E. Meyer, Y. Yun, J.L. Jameson, and J.F. Habener. 1988. Cyclic AMP-responsive DNA-binding protein: Structure based on a cloned placental cDNA. Science 242: $1430-1433$.

Hope, I.A. and K. Struhl. 1987. GCN4, a eukaryotic transcriptional activator protein, binds as a dimer to target DNA. EMBO I. 6: 2781-2784.

Jones, R.H. and N.C. Jones. 1989. Mammalian cAMP-responsive element can activate transcription in yeast and binds a yeast factor(s) that resembles the mammalian transcription factor ATF. Proc. Natl. Acad. Sci. 86: 2176-2180.

Landschulz, W.H., P.F. Johnson, and S.L. McKnight. 1988. The leucine zipper: A hypothetical structure common to a new class of DNA-binding proteins. Science 240: 1759-1764. 
Hai et al.

1989. The DNA-binding domain of the rat liver nuclear protein C/EBP is bipartite. Science 243: 1681-1688.

Lee, K.A.W. and M.R. Green. 1987. A cellular transcription factor E4F1 interacts with an ElA-inducible enhancer and mediates constitutive enhancer function in vitro. EMBO $J$. 6: $1345-1353$.

Lee, K.A.W., T.Y. Hai, L. SivaRaman, B. Thimmappaya, H.C. Hurst, N.C. Jones, and M.R. Green, M.R. 1987. A cellular protein, activating transcription factor, activates transcription of multiple E1A-inducible adenovirus early viral promoters. Proc. Natl. Acad. Sci. 84: 8355-8359.

Lee, K.A.W., J.S. Fink, R.H. Goodman, and M.R. Green. 1989. Distinguishable promoter elements containing ATF-binding sites are involved in transcriptional activation by E1A and cAMP. Mol.Cell. Biol. 9: 4390-4397.

Lin, Y.S. and M.R. Green. 1988. Interaction of a common cellular transcription factor, ATF, with regulatory elements in both E1A- and cyclic AMP-inducible promoters. Proc. Natl. Acad. Sci. 85: 3396-3400.

. 1989. Identification and purification of a Saccharomyces cerevisiae protein with the DNA binding specificity of mammalian activating transcription factor. Proc. Natl. Acad. Sci. 86: 109-113.

Maekawa, T., H. Sakura, C. Kanei-Ishii, T. Sudo, T. Yoshimura, J. Fujisawa, M. Yoshida, and S. Ishii. 1989. Leucine zipper structure of the protein CRE-BP1 binding to the cyclic AMP response element in brain. EMBO J. 8: 2023-2028.

McKnight, S. and R. Tjian. 1986. Transcriptional selectivity of viral genes in mammalian cells. Cell 46: 795-805.

Needleman, S.B. and C.D. Wunsch. 1970. A general method applicable to the search for similarities in the amino acid sequence of two proteins. J. Mol. Biol. 48: 443-453.

Roesler, W.J., G.R. Vandenbark, and R.W. Hanson. 1988. Cyclic AMP and the induction eukaryotic gene transcription. $/$. Biol. Chem. 263: 9063-9066.

Sawadogo, M., M.W. Van Dyke, P.D. Gregor, and R.G. Roeder. 1988. Multiple forms of the human gene-specific transcription factor USF. J. Biol. Chem. 263: 11985-11993.

Singh, H., J.H. LeBowitz, A.S. Baldwin, and P.A. Sharp. 1988. Molecular cloning of an enhancer binding protein: Isolation by screening of an expression library with a recognition site DNA. Cell 52: 415-423.

Turner, R. and R. Tjian. 1989. Leucine repeats and an adjacent DNA-binding domain mediate the formation of functinoal c-Fos and c-Jun heterodimers. Science 243: 1689-1694.

Vinson, C.R., K.L. LaMarco, P.F. Johnson, W.H. Landschulz, and S.L. McKnight. 1988. In situ detection of sequence-specific DNA-binding activity specificed by a recombinant bacteriophage. Genes Dev. 2: 801-806.

Yamamoto, K.K., G.A. Gonzalez, W.H. Biggs, and M.R. Montminy. 1988. Phosphorylation-induced binding and transcriptional efficiency of nuclear factor CREB. Nature 334: 494498. 


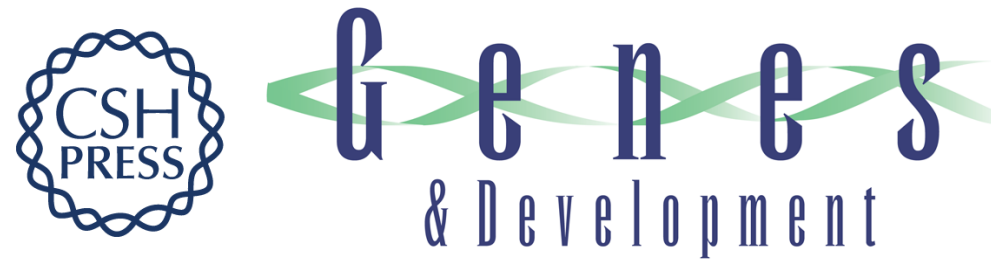

\section{Transcription factor ATF cDNA clones: an extensive family of leucine zipper proteins able to selectively form DNA-binding heterodimers.}

T W Hai, F Liu, W J Coukos, et al.

Genes Dev. 1989, 3:

Access the most recent version at doi:10.1101/gad.3.12b.2083

References This article cites 27 articles, 16 of which can be accessed free at:

http://genesdev.cshlp.org/content/3/12b/2083.full.html\#ref-list-1

License

Email Alerting

Service

Receive free email alerts when new articles cite this article - sign up in the box at the top right corner of the article or click here.

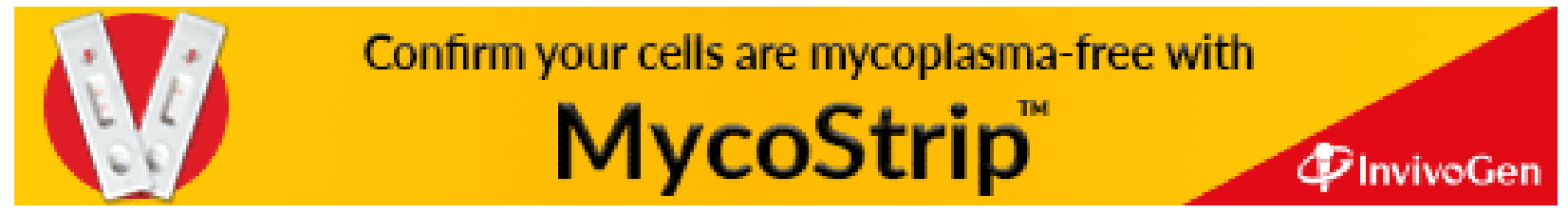

